\title{
Radiotherapy treatment of large esophageal leiomyosarcoma: A case report
}

\author{
SHUANGSHUANG MA ${ }^{1 *}$, WENZHE BU ${ }^{1 *}$, LIJUN WANG ${ }^{2}$, JINPENG LI $^{1}$, \\ CONGCONG SHI ${ }^{3}$, JINLONG SONG ${ }^{1}$ and HUA CHEN ${ }^{1}$
}

\author{
${ }^{1}$ Department of Surgical Oncology (Interventional Therapy), Shandong Cancer Hospital and Institute, \\ Shandong Academy of Medical Sciences, Jinan, Shandong 250117; ${ }^{2}$ Department of Computerized Tomography, \\ Shandong Medical Imaging Research Institute, Jinan, Shandong 250000; ${ }^{3}$ Sixth Ward of \\ Shandong Mental Health Center, Jinan, Shandong 250014, P.R. China
}

Received June 16, 2014; Accepted February 17, 2015

DOI: 10.3892/ol.2015.3065

\begin{abstract}
Leiomyosarcoma of the esophagus is a rare type of tumor, characterized by a malignant phenotype and smooth muscle histology. Previously, barium studies have been used to identify areas of luminal narrowing, expansile intraluminal masses or large intramural masses with ulceration or tracking. Furthermore, endoscopic biopsies appear to be associated with a high false negative rate, particularly in cases where the mucosa is intact. The optimal treatment strategy is surgical resection, while the role of adjuvant radiotherapy and chemotherapy is controversial. In addition, the prognosis of patients with leiomyosarcoma of the esophagus is improved compared with patients suffering from squamous esophageal cancer. The present study described the case of a 48-year-old woman who presented with dysphagia and was diagnosed with a large leiomyosarcoma of the esophagus. The patient was successfully treated with radiotherapy and remains disease-free two years after the completion of treatment. In addition, the present study conducted a review of the relevant literature, reporting previous cases of esophageal leiomyosarcoma and potential strategies for the management of this disease.
\end{abstract}

\section{Introduction}

Squamous cell and adenocarcinoma are the two most common types of esophageal malignancies, whereas esophageal leiomyosarcoma is a rare type of tumor, accounting for $<1 \%$ of all malignant esophageal tumors $(1,2)$. Since the first case of esophageal leiomyosarcoma was reported in 1902 (3), >165

Correspondence to: Professor Jinlong Song, Department of Surgical Oncology (Interventional Therapy), Shandong Cancer Hospital and Institute, Shandong Academy of Medical Sciences, 440 Jiyan Road, Jinan, Shandong 250117, P.R. China

E-mail: jlsong1026@126.com

${ }^{*}$ Contributed equally

Key words: esophagus, leiomyosarcoma, radiotherapy cases of esophageal leiomyosarcoma have been described in the literature (4). Leiomyosarcomas are characterized by slow growth and late metastases and thus, exhibit a better prognosis than squamous cell carcinoma of the esophagus (5). The one-, three- and five-year survival rates of esophageal leiomyosarcoma in the Chinese population are $60.3,42.8$ and $32.1 \%$, respectively (5). The most common symptom observed at diagnosis is progressive dysphagia which occurs in $64.7-90.0 \%$ of esophageal leiomyosarcoma patients. Other symptoms include retrosternal/back pain, weight loss and emesis $(6,7)$. The development of definite treatment strategies is difficult due to the rarity of these tumors. Currently, surgical resection is the first-line treatment (6); however, surgical resection is not suitable for all patients. Although leiomyosarcoma is not sensitive to radiation, radiotherapy can be used to control the tumor effectively by appropriately increasing the radiation dose (8-12). The present study describes a case of leiomyosarcoma of the esophagus that was treated with radiation, rather than surgery. In addition, the current study conducted a review the relevant literature. Written informed consent was obtained from the patient.

\section{Case report}

In May 2009, a 48-year-old woman presented with $6 \mathrm{~kg}$ weight loss in two months and a six-month history of dysphagia to solids, which had been aggravated for two months, at the Shandong Cancer Hospital and Institute (Jinan, China). Computerized tomography (CT) scanning of the chest revealed the presence of a round soft tissue mass with a cross-sectional diameter of $65 \times 40 \mathrm{~mm}$, located at the posterior wall of the esophagus (Fig. 1A). In addition, upper esophageal dilation was observed, with a diameter of $\sim 90 \mathrm{~mm}$ between the upper and lower esophagus. Furthermore, barium studies and esophagoscopy demonstrated that the lesion was located in the lower esophagus, $15-25 \mathrm{~cm}$ from the upper gum margin. A biopsy specimen of the mass was obtained during esophagoscopy and used to determine a diagnosis of poorly differentiated esophageal leiomyosarcoma. Histological examination of the tumor revealed markedly anaplastic spindle cells with elongated nuclei. On immunohistochemistry, the tumor cells were positive 

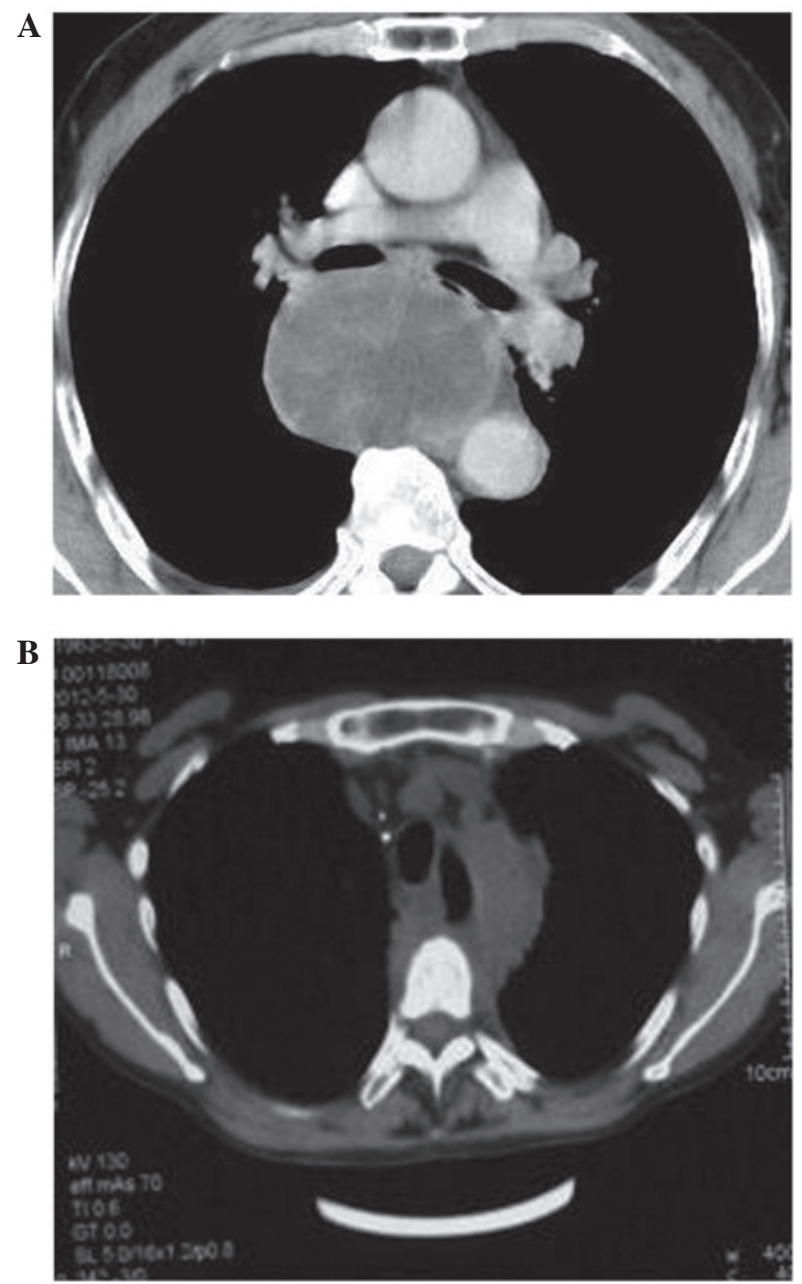

Figure 1. (A) Computed tomography (CT) scan performed prior to radiotherapy, revealing a parenchyma mass in the esophagus that was oppressing the trachea. (B) CT scan performed six months after radiotherapy, indicating that the tumor reduced in size.

for vimentin and smooth muscle actin, and negative for cytokeratin, epithelial membrane antigen, S-100 and C-kit. Due to the large tumor volume and infiltration of the upper esophagus resection was not performed. The patient subsequently received intensity-modulated radiotherapy (IMRT) (60 Gy/30 fractions) at the Shandong Cancer Hospital and Institute between June 1 and July 20, 2009.

After one month of IMRT, a CT scan revealed that the lesion was not significantly reduced in size; however, the symptom of dysphagia was improved. Six months later, the CT scans indicated that the tumor was significantly reduced in size (Fig. 1B); thus, the patient was able to consume a normal diet, resulting in a weight increase of $5 \mathrm{~kg}$. Follow-up examinations were performed for two years, which revealed that the tumor had significantly decreased in size, and at the final follow-up the patient was asymptomatic with no identified tumor recurrences.

\section{Discussion}

Esophageal leiomyosarcoma is a rare type of malignant tumor, accounting for $<1 \%$ of all esophageal malignancies (13). Dysphagia is the predominant clinical manifestation in patients suffering from an esophageal leiomyosarcoma (4), which occurs in $64.7-90.0 \%$ of patients (7). Other symptoms include retrosternal/back pain, weight loss and emesis. Tumor features include infiltration of the esophageal wall and expansive growth; therefore, foreign invasion is less commonly identified (7). Furthermore, previous studies have reported that the obstructive symptoms are proportional to tumor size; therefore, early symptoms of obstruction are not evident and the majority of patients presented significant obstructive symptoms when the tumor was large $(14,15)$. In addition, the preoperative misdiagnosis rate is as high as $82 \%$. The majority of cases are finally diagnosed by performing an esophageal biopsy and immunohistochemical analyses (1). Additionally, esophagogram, computed tomography (CT), glucose positron emission tomography/CT and endoscopic ultrasonography may also aid diagnosis (16). However, distinguishing leiomyosarcomas from other esophageal neoplasms remains difficult (17). Stelow et al (18) recently reported that endoscopic ultrasound-guided fine-needle aspiration (EUS-FNA) may present a safe and accurate method for distinguishing the leiomyosarcoma from other esophageal tumors and may be used to guide therapy. In the current study, leiomyosarcoma was diagnosed by EUS-FNA.

The detection rate of esophageal leiomyosarcoma using barium esophagography has been reported to be 64-68\%. The results of barium studies commonly reveal large intramural masses with a marked exophytic component and often contain areas of ulceration or tracking (7). Using endoscopic sonography, previous studies have identified esophageal leiomyosarcomas as well-defined hyperechoic masses arising from the muscular layer of the esophageal wall (19). Additionally, these tumors may be recognized on angiography images as hypervascular masses with tumor vessels, dilated vascular channels or venous lakes, and early venous drainage. The development of immunohistochemical detection methods helped to improve the clinical understanding of esophageal leiomyosarcomas, as well as differentiate between esophageal stromal tumors and leiomyosarcomas.

Surgery is a safe and effective procedure for the treatment of esophageal leiomyosarcomas. As lymph node metastasis is relatively rare in this disease, esophageal leiomyosarcoma surgery is associated with longer five-year survival rates and, thus, improved prognosis compared with the surgical treatment of esophageal cancer (20). Radiotherapy may be used as adjuvant treatment, in cases where the risk of local recurrence is considered to be high, for example, in high-grade tumors, or when limb preservation is important. Limited surgery may be used to avoid amputation or the excessive loss of tissue, followed by radiotherapy in order to sterilize the remaining malignant cells (21-24). It has been reported that completely resected lowgrade soft-tissue tumors do not require additional treatment (25). In addition, radiotherapy alone may be recommended in cases where a tumor is inoperable, recurrent or metastatic disease has occurred or for palliation (26). Due to the large volume of the tumor and infiltration of the esophagus, the patient of the current study underwent radiotherapy alone and surgery was not performed. Since leiomyosarcomas exhibit poor sensitivity to radiation, the radiation dose should be increased from the conventional dose of 60-65 Gy, which is used to treat other tumor types (23) to $75 \mathrm{~Gy}$. Therefore, the current patient underwent IMRT. Considering the large size of the tumor and the low rate 
of metastasis to the lymph node, the clinical target volume did not include the lymphatic drainage area.

Leiomyosarcoma is typically associated with a good prognosis due to its slow growth and the late occurrence of metastasis (27). However, due to its rarity, it is difficult to determine a pre-operative diagnosis and appropriate treatment strategy for cases of esophageal leiomyosarcoma. In previous years, leiomyosarcoma was not considered to be sensitive to radiation; therefore, radical radiotherapy treatment was rarely administered and a limited number of reports regarding the use of radiotherapy in the treatment of leiomyosarcoma exist in the literature $(6,24,28)$. Although leiomyosarcoma has poor sensitivity to radiation, the tumor may be effectively controlled by increasing the radiation dose appropriately. As demonstrated in the present study, three-dimensional conformal radiotherapy and IMRT may exhibit a sufficient protective effect on vital organs adjacent to the tumor and may be an effective topical treatment strategy for patients unable to undergo surgery.

In conclusion, esophageal leiomyosarcoma has an excellent prognosis, and radical resection may achieve acceptable results. However, surgery may not be suitable, depending on the location of the tumor or the occurrence of distant metastases. Previously, leiomyosarcoma was considered to be insensitive to radiation and thus, radical radiotherapy treatment was rarely administered and only a limited number of reports regarding the use of radiotherapy in the treatment of leiomyosarcoma exist in the literature. Although leiomyosarcoma exhibits poor sensitivity to radiation, the tumor may be effectively controlled by increasing the radiation dose appropriately. In the present study, surgery was unsuitable for the patient due to the size and location of tumor. However, the tumor was successfully treated with radiotherapy and the patient has an excellent prognosis. Radiotherapy should also be recommended for the treatment of metastatic tumors to prolong survival in cases exhibiting extensive or unresectable metastases.

\section{References}

1. Choh JH, Khazei AH and Ihm HJ: Leiomyosarcoma of the esophagus: report of a case and review of the literature. J Surg Oncol 32: 223-226, 1986.

2. Weinstein EC, Kim YS, Young GJ and Kasimian D: Leiomyosarcoma of the esophagus. Mil Med 153: 206-209, 1988.

3. Howard WT: Primary sarcoma of the esophagus and stomach. JAMA 38: 392-399, 1902.

4. Hatch GF III, Wertheimer-Hatch L, Hatch KF, et al: Tumors of the esophagus. World J Surg 24: 401-411, 2000.

5. Koga $\mathrm{H}$, Iida M, Suekane $\mathrm{H}$, et al: Rapidly growing esophageal leiomyosarcoma: case report and review of the literature. Abdom Imaging 20: 15-19, 1995.

6. Rocco G, Trastek VF, Deschamps C, et al: Leiomyosarcoma of the esophagus: results of surgical treatment. Ann Thorac Surg 66: 894-896, discussion 897, 1998.

7. Levine MS, Buck JL, Pantongrag-Brown L, et al: Leiomyosarcoma of the esophagus: radiographic findings in 10 patients. AJR Am J Roentgenol 167: 27-32, 1996.
8. Pramesh CS, Pantvaidya GH, Moonim MT, et al: Leiomyosarcoma of the esophagus. Dis Esophagus 16: 142-144, 2003.

9. Gao Y, Wang L and Zhang D: Surgical treatment of esophageal leiomyosarcoma: a review of the literature and report of 11 cases. Zhonghua Zhong Liu Za Zhi 21: 470-472, 1999 (In Chinese).

10. Shiraishi M, Takahashi T, Yamashiro M, et al: A report of leiomyosarcoma of the esophagus. Nihon Ronen Igakkai Zasshi 32: 286-291, 1995 (In Japanese).

11. Mutrie CJ, Donahue DM, Wain JC, et al: Esophageal leiomyoma: a 40-year experience. Ann Thorac Surg 79: 1122-1125, 2005.

12. Wang WX, Gaurav D, Wen L, et al: Pediatric esophageal leiomyosarcoma: a case report. J Pediatr Surg 46:1646-1650, 2011.

13. Galandiuk S, Hermann RE, Cosgrove DM and Gassman JJ: Cancer of the esophagus. The Cleveland Clinic experience. Ann Surg 203: 101-108, 1986.

14. Wang Q, Ye T, Jiang W and Lin ZW: Video-assisted thoracoscopic surgery in the treatment of esophageal leiomyoma: a report of 39 cases. Zhonghua Wei Chang Wai Ke Za Zhi 13: $145-147,2010$.

15. Lee LS, Singhal S, Brinster CJ, et al: Current management of esophageal leiomyoma. J Am Coll Surg 198: 136-146, 2004.

16. Kimura H, Konishi K, Kawamura T, et al: Smooth muscle tumors of the esophagus: clinicopathological findings in six patients. Dis Esophagus 12: 77-81, 1999.

17. Aimoto T, Sasajima K, Kyono S, et al: Leiomyosarcoma of the esophagus: report of a case and preoperative evaluation by CT scan, endoscopic ultrasonography and angiography. Gastroenterol Jpn 27: 773-779, 1992.

18. Stelow EB, Jones DR and Shami VM: Esophageal leiomyosarcoma diagnosed by endoscopic ultrasound-guided fine-needle aspiration. Diagn Cytopathol 35: 167-170, 2007.

19. Puli SR, Reddy JB, Bechtold ML, et al: Staging accuracy of esophageal cancer by endoscopic ultrasound: a meta-analysis and systematic review. World J Gastroenterol 14: 1479-1490, 2008.

20. Takayama T, Kato H, Tachimori Y, et al: Treatment of rupture of a liver metastasis from esophageal leiomyosarcoma. Jpn J Clin Oncol 26:248-251, 1996

21. Franklin GO, Antler AS, Thelmo WL and Rosenthal WS: Esophageal leiomyosarcoma. NY State J Med 82: 1100-1103, 1982.

22. Lin SH, Wang L, Myles B, et al: Propensity score-based comparison of long-term outcomes with 3-dimensional conformal radiotherapy vs intensity-modulated radiotherapy for esophageal cancer. Int J Radiat Oncol Biol Phys 84: 1078-1085, 2012.

23. Wang J, Han C, Li X N, et al: Short-term efficacy of intensity-modulated radiotherapy on esophageal carcinoma. Ai Zheng 28: 1138-1142, 2009 (In Chinese).

24. Perch SJ, Soffen EM, Whittington R and Brooks JJ: Esophageal sarcomas. J Surg Oncol 48: 194-198, 1991.

25. Markhede G, Angervall L and Sterner B: A multivariate analysis of the prognosis after surgical treatment of malignant soft-tissue tumours. Cancer 49: 1721-1733, 1982.

26. Athanasoulis CA and Aral IM: Leiomyosarcoma of the esophagus. Gastroenterology 54: 271-274, 1968.

27. Zhang BH, Zhang HT and Wang YG: Esophageal leiomyosarcoma: clinical analysis and surgical treatment of 12 cases. Dis Esophagus 27: 547-551, 2014.

28. Futuri S, Donohoe K, Spaccavento C and Yudelman I: Rectal leiomyosarcoma: a rare and long-term complication of radiation therapy. BMJ Case Rep: Oct 14, 2014 (Epub ahead of print). 